\title{
Study of Dickkopf-1 (DKK-1) in patients with chronic viral hepatitis C-related liver cirrhosis with and without hepatocellular carcinoma
}

\author{
Mona Kamal Eldeeb', Gehan Mahmoud Magour', Rania Nabil Bedair', Mohammed Mahmoud Shamseya', \\ Mennatallah Abdelfattah Hammouda² \\ 'Medical Research Institute, Alexandria University, Egypt \\ 2Pharos University in Alexandria, Egypt
}

\begin{abstract}
Aim of the study: Dickkopf-1 (DKK-1) is a secreted protein which acts as an inhibitor of Wnt/ $\beta$-catenin signaling. DKK-1 was found to be a helpful biomarker for many cancers including hepatocellular carcinoma (HCC). HCC is multifactorial in origin and its main etiology in Egypt is attributed to chronic hepatitis C virus (HCV) infection. Objectives: To assess the serum level and diagnostic performance of DKK-1 and $\alpha$-fetoprotein (AFP) in Egyptian patients with chronic HCV-related liver cirrhosis with and without HCC.

Material and methods: 80 subjects were divided into: a control group (group I, 20 healthy volunteers) and two patient groups: group II (HCV with liver cirrhosis, 30 patients), and group III, (HCV-related liver cirrhosis with HCC, 30 patients). Thorough physical examination, triphasic computed tomography, calculation of Child-Pugh score, laboratory investigations (complete blood picture, liver profile, hepatitis B surface antigen, anti-HCV antibodies, AFP (chemiluminometry) and DKK-1 (ELISA) were performed.

Results: There was a significant decrease in DKK-1 level in HCV patients with liver cirrhosis (group II) and HCV patients with HCC (group III) compared to the control group (group I) $(p<0.001)$. However, there was a significant increase in DKK-1 level in HCV patients with HCC (group III) compared to HCV patients with liver cirrhosis (group II) $(p<0.033)$. The ROC curve showed that DKK-1 has less sensitivity but higher specificity in HCV patients with HCC (group III) compared with HCV patients with liver cirrhosis (group II).

Conclusions: The combination of DKK-1 and AFP could further improve the diagnostic accuracy of HCV-related cirrhosis with or without HCC.
\end{abstract}

Key words: hepatocellular carcinoma, hepatitis C virus, Dickkopf-1, $\alpha$-fetoprotein.

\section{Address for correspondence}

Dr. Mona Kamal Eldeeb, Medical Research Institute, Alexandria University, 165 El-Horreya Avenue, El-Hadra POB: 21561, Alexandria, Egypt, e-mail: mona.eldeeb@alexu.edu.eg

\section{Introduction}

Dickkopf-1 (DKK-1) belongs to the DKK family which encodes secreted proteins consisting of four main members (DKK 1-4) [1]. It is a plasma protein with two cysteine rich regions [2]. It was shown that it was implicated in the control of various physiological and pathological processes, including head formation during embryonic development, osteoclastogenesis, and tumor cell related processes (proliferation, survival, migration and invasion) [1-3]. It also plays a role in negative regulation of the Wnt signaling pathway. This Wnt $/ \beta$-catenin pathway regulates gene transcription, cell proliferation and differentiation and can lead to tumor formation [4]. DKK-1 has a controversial role in tumor biology, where it is shown to be either an inhibitor or promotor of tumor progression and metastasis $[5,6]$.

DKK-1 was found to be overexpressed in nonsmall cell lung cancer [5], esophageal squamous cell 
carcinomas [6] and kidney cancers [7]. However, low expression was found in human colon tumors [8].

Hepatocellular carcinoma (HCC) represents the fifth most common neoplasm and the third cause of cancerrelated mortality worldwide. HCC is multifactorial in origin and its main etiology in Egypt is attributed to chronic hepatitis $\mathrm{C}$ viral (HCV) infection [9]. Although some HCCs do arise in the absence of cirrhosis, several steps have been implicated in the carcinogenesis of HCV-associated HCC involving upregulation of inflammatory cytokines and induction of oxidative stress from the process of chronic hepatitis, fibrosis, liver regeneration, and, ultimately, the development of cirrhosis [10].

Elevated DKK-1 levels were found in serum of patients with hepatocellular carcinoma (HCC) in addition to increased expressions in hepatic tissues of HCC patients. Thus its overexpression may not only help the formation and growth of tumors but also may initiate the cell invasion and metastasis in vitro and in vivo, whereas knockdown of DKK-1 significantly reduces both migratory and invasive abilities of HCC cells [3].

Currently, diagnosis of HCC is done by radiological means (triphasic computed tomography and/or magnetic resonance imaging) and tumor markers, mainly AFP [11].

The present work aimed at evaluating the serum level of DKK-1 in a cohort of Egyptian patients with HCV-related liver cirrhosis with or without hepatocellular carcinoma, in order to determine its role in occurrence of both HCV liver cirrhosis alone and HCC on top of HCV liver cirrhosis.

\section{Material and methods}

After the approval of the Ethics Committee of the Medical Research Institute (February 2015), eighty Egyptian subjects were included in this case control study. Informed consent was taken from each participant. They were divided into three main groups. The control group (group I) included twenty adult apparently healthy volunteers. Cirrhotic HCV patients without HCC (group II) included thirty patients. The group of cirrhotic HCV patients with HCC (group III) included thirty patients. All patients included in the present study were attending the Hepatology Unit, Medical Research Institute, Alexandria University, Egypt. Patients with positive hepatitis B surface antigen (HBsAg +ve), rheumatoid arthritis, other malignant conditions and patients receiving chemotherapeutic agents or after HCC tumor resection were excluded from the present study.

In all the studied patients thorough physical examination and triphasic computed tomography were done. In all cirrhotic HCV patients with or without HCC using gadolinium as contrast medium HCC patients were diagnosed through enhancement of the focal lesion in the arterial phase with washout in the venous phase, along with the calculation of the ChildPugh (CP) score. Laboratory investigations done included the determination of complete blood picture, measurement of the serum levels of bilirubin (total and direct) and albumin and aspartate aminotransferase (AST), alanine aminotransferase (ALT), $\gamma$-glutamyl transferase (GGT) and alkaline phosphatase (ALP) activities (Table 1). Sero-detection of HBsAg and HCV antibodies were done to rule out HBV and confirm $\mathrm{HCV}$ infection, in addition to estimation of serum AFP by the chemiluminometric method and DKK-1 level by ELISA technique [12].

\section{Statistical analysis}

Statistical analysis was done using IBM SPSS Statistics 20.0 (Statistical Package of Social Sciences, Chicago, USA) [13] for quantitative variables. The Kolmogorov-Smirnov test for normality was used to test for the degree of deviation from normal distribution across all variables. The data of the variables were summarized in the form of: the mean as a measure of central tendency and the standard deviation (SD) as a measure of dispersion for normally distributed data and as median and range for abnormally distributed data. The oneway ANOVA parametric test was used to compare the means for quantitative data of more than two independent groups normally distributed. The Kruskal-Wallis non-parametric test was used for testing equality of population medians among more than two independent groups. The Mann-Whitney non-parametric test was used for testing equality of population medians between two independent groups. Pearson and Spearman correlation coefficients were used to calculate the correlation between variables. $P<0.05$ was considered significant. The ROC curve was drawn by sensitivity on $\mathrm{y}$-axis and 1-specificity on the $\mathrm{x}$-axis. The highest Youden's index calculated gave the best cutoff. The greater area under the curve was the best.

\section{Results}

In the present study no significant difference was found in age or sex between the studied groups. The control group (group I) included twenty apparently healthy volunteers (11 males and 9 females); their mean age was $53.20 \pm 9.61$ years. Patients with chronic HCV-related liver cirrhosis without HCC (group II) included thirty patients (21 males and 9 females); their mean age was $58.0 \pm 7.2$ years. Patients with chronic 
Table 1. Statistically significant differences of laboratory tests performed on the studied groups

\begin{tabular}{|c|c|c|c|c|c|c|c|}
\hline \multirow[t]{2}{*}{ Parameter } & & \multicolumn{3}{|c|}{ Group } & \multirow[t]{2}{*}{$p 1$} & \multirow[t]{2}{*}{ p2 } & \multirow[t]{2}{*}{ p3 } \\
\hline & & $\begin{array}{l}\text { Group I } \\
\text { Control } \\
(n=20)\end{array}$ & $\begin{array}{l}\text { Group II } \\
\text { Liver cirrhosis patients } \\
(n=30)\end{array}$ & $\begin{array}{l}\text { Group III } \\
\text { HCC patients } \\
(n=30)\end{array}$ & & & \\
\hline \multirow[t]{2}{*}{ Total bilirubin (mg/dl) } & Min.-Max. & $0.8-1.0$ & $1.0-31.5$ & $0.4-20.6$ & \multirow[t]{2}{*}{$0.003 *$} & \multirow[t]{2}{*}{$0.001^{*}$} & \multirow[t]{2}{*}{0.185} \\
\hline & Median & 0.9 & 2.9 & 3.1 & & & \\
\hline \multirow[t]{2}{*}{ Direct bilirubin (mg/dl) } & Min.-Max. & $0.1-0.2$ & $0.3-29.4$ & $0.3-13.8$ & \multirow[t]{2}{*}{$0.003^{*}$} & \multirow[t]{2}{*}{$0.001^{*}$} & \multirow[t]{2}{*}{$0.047^{*}$} \\
\hline & Median & 0.15 & 1.50 & 1.65 & & & \\
\hline \multirow[t]{2}{*}{ Albumin (g/dl) } & Min.-Max. & $3.5-5.1$ & $1.2-4.10$ & $1.7-3.10$ & \multirow[t]{2}{*}{$0.001 *$} & \multirow[t]{2}{*}{$0.001^{*}$} & \multirow[t]{2}{*}{0.524} \\
\hline & Median & 4.0 & 2.40 & 2.10 & & & \\
\hline \multirow[t]{2}{*}{ AST (U/I) } & Min.-Max. & $15.0-35.0$ & $19.0-311.0$ & $22.0-418.0$ & \multirow[t]{2}{*}{$0.001^{*}$} & \multirow[t]{2}{*}{$0.001^{*}$} & \multirow[t]{2}{*}{$0.003^{*}$} \\
\hline & Median & 29.50 & 49.50 & 66.50 & & & \\
\hline \multirow[t]{2}{*}{ ALT (U/l) } & Min.-Max. & $12.0-31.0$ & $12.0-121.0$ & $14.0-192.0$ & \multirow[t]{2}{*}{$0.001^{*}$} & \multirow[t]{2}{*}{$0.001^{*}$} & \multirow[t]{2}{*}{$0.026^{*}$} \\
\hline & Median & 21.0 & 22.0 & 33.5 & & & \\
\hline \multirow[t]{2}{*}{$\operatorname{ALP}(\mathrm{U} / \mathrm{I})$} & Min.-Max. & $54.0-92.0$ & $71.0-235.0$ & $85.0-402.0$ & \multirow[t]{2}{*}{$<0.001^{* *}$} & \multirow[t]{2}{*}{$<0.001 * *$} & \multirow[t]{2}{*}{$<0.001 * *$} \\
\hline & Median & 83.0 & 117.0 & 182.0 & & & \\
\hline \multirow[t]{2}{*}{ GGT (U/I) } & Min.-Max. & $16.0-32.0$ & $26.0-126.0$ & $49.0-462.0$ & \multirow[t]{2}{*}{$<0.001^{* *}$} & \multirow[t]{2}{*}{$<0.001^{* *}$} & \multirow[t]{2}{*}{$<0.001^{* *}$} \\
\hline & Median & 22.0 & 50.0 & 91.50 & & & \\
\hline \multirow[t]{2}{*}{ AFP (ng/ml) } & Min.-Max. & $0.98-3.36$ & $1.56-12015.0$ & $2.31-22000.0$ & \multirow[t]{2}{*}{$<0.001^{* *}$} & $<0.001^{* *}$ & $0.001^{*}$ \\
\hline & Median & 2.0 & 3.9 & 101.1 & & & \\
\hline DKK-1 (pg/ml) & Min.-Max. & 658.39-3061.0 & $14.25-1674.8$ & $43.95-3660.5$ & $<0.001^{* *}$ & $<0.001^{* *}$ & $0.033^{*}$ \\
\hline & Median & 1598.4 & 264.5 & 553.1 & & & \\
\hline Platelets $\left(10^{3} / \mu \mathrm{l}\right)$ & Mean $\pm S D$ & $263.4 \pm 50.7$ & $101.7 \pm 47.6$ & $114.9 \pm 60.1$ & $0.001 *$ & $0.001^{*}$ & 0.185 \\
\hline
\end{tabular}

p1 - significant difference between group I and group II

p2 - significant difference between group I and group III

p3 - significant difference between group II and group III

${ }^{*} p<0.05$ (significant), ${ }^{* *} p<0.001$ (very highly significant)

HCV-related liver cirrhosis with HCC (group III) included thirty patients (23 males and 7 females); their mean age was $56.7 \pm 6.1$ years.

Triphasic CT was done in patients with chronic HCV-related liver cirrhosis with HCC (group III). 15 out of $30(50 \%)$ had one lesion, 8 out of $30(26.7 \%)$ had two lesions and 7 out of 30 (23.3\%) had three or more lesions.

In the present study Child-Pugh (CP) classification was done in patients with chronic HCV-related liver cirrhosis without HCC (group II). 2 out of 30 were classified as A, 9 out of 30 were classified as B and 19 out of 30 were classified as C. In patients with chronic HCV-related liver cirrhosis with HCC (group III), it was found that 9 out of 30 were classified as B and 21 out of 30 were classified as C.

In the current study the median value of serum DKK-1 in the control group (group I) was 1598.4 $\mathrm{pg} / \mathrm{ml}$ (ranging from 658.39 to $3061.0 \mathrm{pg} / \mathrm{ml}$ ) (Fig. 1).

There was a significantly lower DKK-1 level in patients with chronic HCV-related liver cirrhosis without

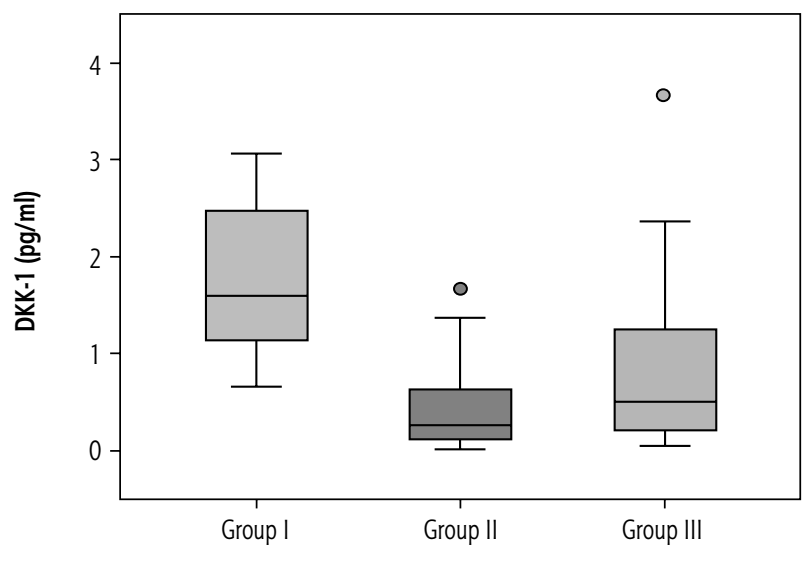

Fig. 1. Box plot presentation of Dickkopf-1 (DKK-1) $(\mathrm{pg} / \mathrm{ml})$ in the studied groups. The box plot represents the interquartile range from $25^{\text {th }}$ to the $75^{\text {th }}$ percentiles. The whiskers below and above the box represents 10-90 percentiles. The line across each box represents the median value

HCC (group II) compared to the control group (group I) $(p<0.001)$ and there was a significantly lower DKK-1 level in patients with chronic HCV-related liver cir- 


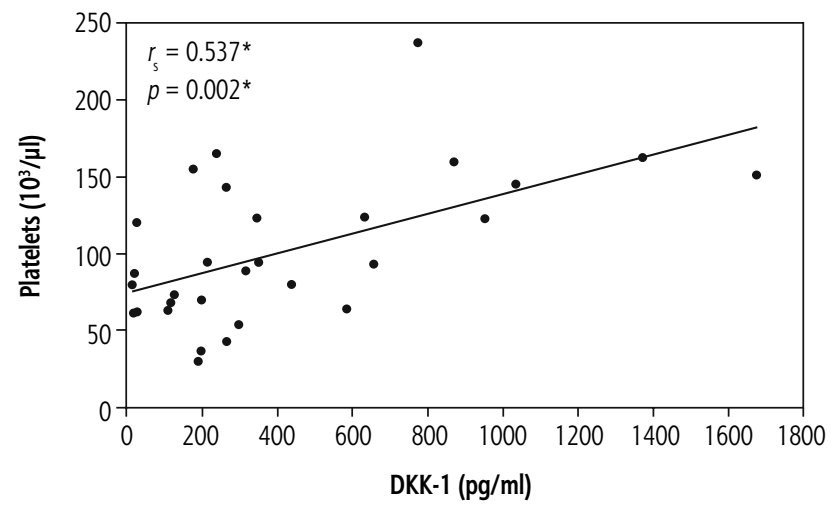

Fig. 2. Significant correlations between DKK-1 and platelets in liver cirrhosis group (group II)

rhosis with HCC (group III) compared to the control group (group I) $(p<0.001)$.

In the current work there was a significant positive correlation of DKK-1 with platelets in patients with chronic HCV-related liver cirrhosis without HCC (group II) $\left(r_{\mathrm{s}}=0.537, p=0.002\right)$ (Fig. 2) and in patients with chronic HCV-related liver cirrhosis with HCC (group III) $\left(r_{\mathrm{s}}=0.540, p=0.002\right)$ (Fig. 3). In addition, patients with chronic HCV-related liver cirrhosis without HCC (group II) and patients with chronic HCV-related liver cirrhosis with HCC (group III) showed thrombocytopenia.

A significantly higher level of DKK-1 was found in patients with chronic HCV-related liver cirrhosis with HCC (group III) compared to patients with chronic HCV-related liver cirrhosis without HCC (group II) $(p=0.033)$.

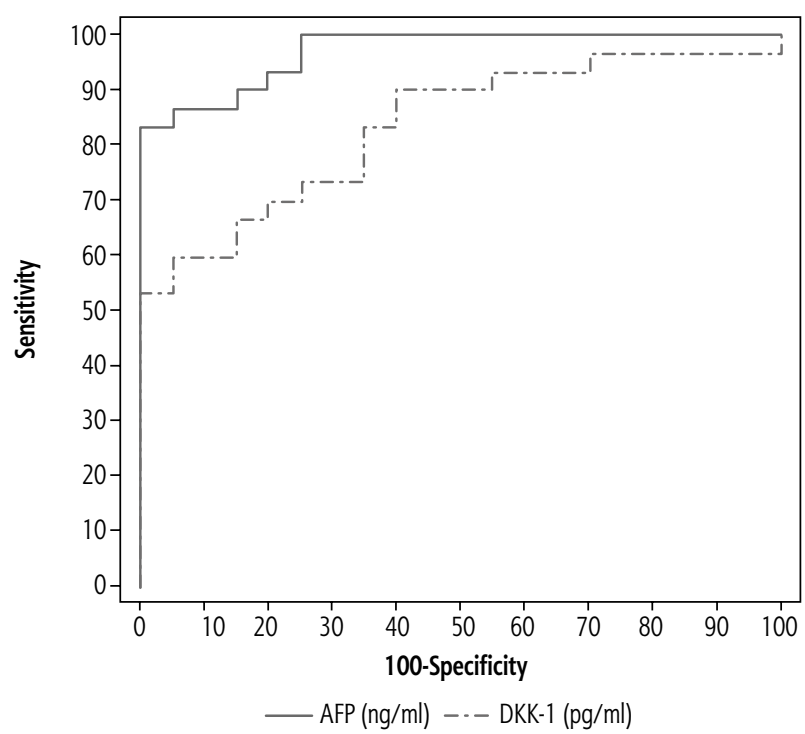

Fig. 4. Receiver operator characteristic curve (ROC) of $\alpha$-fetoprotein (AFP) and Dickkopf-1 (DKK-1) in the control group (group I) and patients with chronic viral hepatitis C-related liver cirrhosis with HCC (group III)

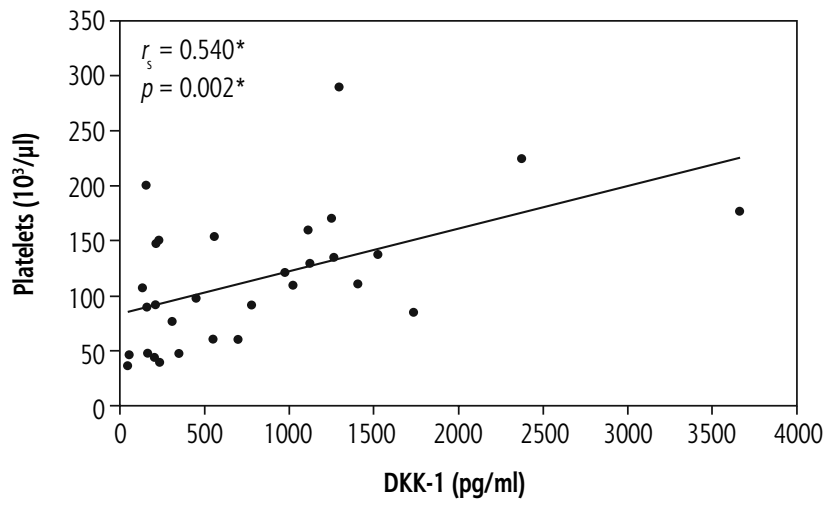

Fig. 3. Significant correlations between DKK-1 and platelets in hepatocellular carcinoma group (group III)

The was no statistically significant difference $(p=0.08)$ in DKK-1 levels between cirrhotic HCV patients class $\mathrm{A}$ and $\mathrm{B}$ compared to cirrhotic HCV patients $\mathrm{CP}$ class $\mathrm{C}$, or between cirrhotic HCV patients with HCC class B compared to those class $\mathrm{C}$ of the same group $(p=0.09)$.

The ROC curve in the control group (group I) and patients with chronic HCV-related liver cirrhosis with HCC (group III) showed sensitivity AFP of $83.33 \%$, specificity of $100 \%$, positive predictive value (PPV) of $100 \%$, negative predictive value (NPV) of $80 \%$, cut-off value of $>3.36 \mathrm{ng} / \mathrm{ml}$, area under the curve (AUC) of 0.970 . DKK-1 showed sensitivity of $60 \%$, specificity of $95 \%$, PPV of $94.7 \%$, NPV of $61.3 \%$, cut-off value of $\leq 774.63 \mathrm{pg} / \mathrm{ml}$, AUC of 0.835 (Fig. 4).

The ROC curve in our study in patients with chronic HCV-related liver cirrhosis without HCC (group II) and patients with chronic HCV-related liver cirrhosis with HCC (group III) showed AFP sensitivity of 76.67\%, specificity of $77.42 \%$, PPV of $75.9 \%$, NPV of $74.2 \%$, cut-off value of $>6.99 \mathrm{ng} / \mathrm{ml}$, AUC of 0.791 . DKK-1 showed sensitivity of $40 \%$, specificity of $90 \%$, PPV of $80 \%$, NPV of $60 \%$, cut-off value of $>952.33 \mathrm{pg} / \mathrm{ml}$, AUC of 0.660 (Fig. 5).

\section{Discussion}

Dickkopf-1 is a secretory antagonist of the canonical Wnt signaling pathway which is hardly expressed in normal human adult tissues except in placental and embryonic tissues [14]. DKK-1 was considered as a diagnostic and prognostic biomarker in many studies on multiple human cancers [14-16]. DKK-1 was reported to have a role in HCC cell migration and invasion [17].

Hepatocellular carcinoma is a disease with poor prognosis due to its insidious onset and late presentation at diagnosis. Thus early detection of HCC with effective screening systems may result in more effective treatment and extend patient survival [18]. 
Alpha-fetoprotein is the first serologic assay for HCC detection [19]. However, many patients with benign liver diseases, such as chronic hepatitis, liver cirrhosis and gastrointestinal cancer, also have elevated serum AFP levels; thus additional biomarkers are needed as a complement to AFP [20]. Therefore we studied DKK-1 as a diagnostic serum marker in HCC patients and compared its diagnostic performance with AFP.

The healthy control group was selected as nonfibrotic patients as the pro-fibrogenic role of hepatocytes in schistosomiasis-induced liver fibrosis is dependent on the Wnt signaling pathway [21]. Figure 1 shows the level of serum DKK-1 in the studied groups, where there was a difference in the median serum level in the present study control group and other studies' healthy control group (115 pg/ml and $1201.46 \mathrm{pg} / \mathrm{ml}$ respectively) $[22,23]$. These differences could be attributed to differences in the groups chosen regarding age, sex, ethnicity and variability of kits of the reagent used.

Opposite to the present study when comparing DKK-1 serum level between HCV cirrhotic patients without HCC and controls, Liu et al. [24] found no significant difference between HCV infection and level of DKK-1 expression and reported that HCV core protein activates $\mathrm{Wnt} / \beta$-catenin signaling with over-expression of canonical Wnt ligands and moderate repression of Wnt antagonists including DKK-1.

The significant decrease in DKK-1 levels in HCV cirrhotic patients with HCC and controls was in agreement with Ahmed et al. [25], who explained that DKK-1's role as an antagonist of Wnt signaling could be lost in cancer and the induction of DKK-1 expression is lost as epigenetic silencing of DKK- 1 .

Huang et al. [26] found that levels of DKK-1 in serum were significantly higher in patients with HCC than in controls and documented that overexpression of DKK-1 plays a role in HCC cell invasion and metastasis and thus it may be used in liver cancer as a potential molecular therapeutic target.

Kim et al. [12] studied DKK-1 gene expression and found high expression of DKK-1 mRNA in addition to increased DKK-1 protein secretion in culture media of cell lines of HCC in comparison to the control. It was found that the ability of DKK-1 to inhibit Wnt signaling was abolished in HCC, most likely due to genetic alterations disrupting the central multi-protein complex that controls $\beta$-catenin stability. However, the exact role of DKK-1 played in migration and invasion was unclear; thus further studies are needed to explain the molecular mechanism of this controversial phenomenon.

Voorzanger-Rousselot et al. [27] found that platelets were a major source of circulating DKK-1 and concluded that DKK-1 could be stored in platelets and

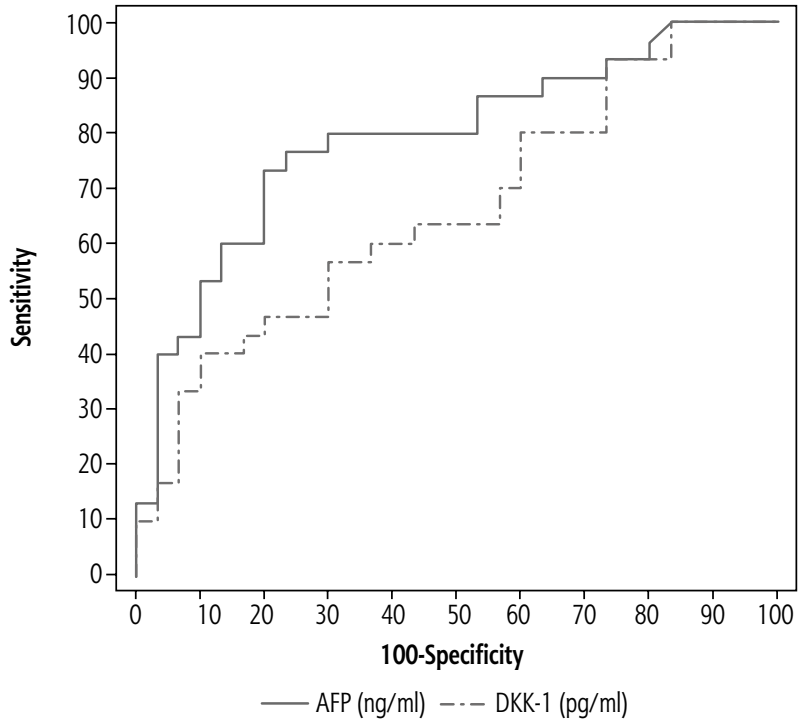

Fig. 5. Receiver operator characteristic curve (ROC) of $\alpha$-fetoprotein (AFP) and Dickkopf-1 (DKK-1) in patients with chronic viral hepatitis C-related liver cirrhosis without HCC group (group II) and patients with chronic viral hepatitis C-related liver cirrhosis with HCC (group III)

released in the serum during blood coagulation in patients with multiple myeloma and this could explain the positive correlations found in the present study between DKK-1 and platelets in both HCV cirrhotic patients with and without HCC. Also the thrombocytopenia found in both groups could explain the decrease in DKK-1 serum levels in them.

Fouad et al. [28] found in agreement with our study significantly higher serum DKK-1 level in HCV cirrhotic patients with HCC than HCV cirrhotic patients without HCC, which could explain why DKK-1 might act as a tumor suppressor.

No significant difference was found between HCC patients and chronic HCV groups in the study conducted by Shen et al. [29], who explained this by the presence of accumulation of $\beta$-catenin in the cytoplasm through $\beta$-catenin/T-cell factor because of the activity of $\mathrm{HCV}$ virus. Also unexpected higher concentrations of serum DKK-1 were found in HCC patients without cirrhosis than in those with cirrhosis.

Shi et al. [30] showed that DKK-1 is not specific for HCC diagnosis and reported that serum DKK-1 was also elevated in patients with intrahepatic cholangiocarcinoma.

Tung et al. [16] found a stepwise-like increase in both serum DKK-1 levels and in its transcript in human HCCs. Also its transcript levels were associated with more aggressive tumor behavior.

Qin et al. [31] showed that in HCC patients, DKK-1 has been used to assess HCC prognosis and overexpression of DKK-1 was found to be related to poor survival outcomes. One study in Chinese HCC patients 
revealed that those with higher DKK-1 levels had both a lower 5-year overall survival rate and a lower disease-free survival rate, compared with patients with lower levels of DKK-1 [32].

In the present study ROC curve analysis was performed to show the diagnostic performance of serum AFP and DKK- 1 and the AUCs were calculated to determine the best cut off-value, sensitivity and specificity of each marker in diagnosing HCC.

Our ROC curve results were different from the results of two other studies where the diagnostic performance of DKK-1 and AFP were as follows: DKK-1 showed sensitivity of $76.0 \%$ and $79.78 \%$, specificity of $93.8 \%$ and $93.07 \%$, PPV of $91.9 \%$ and 91.03 , NPV of $80.8 \%$ and $83.93 \%$ and AUC of 0.877 and 0.902 , respectively. AFP showed sensitivity of $50.0 \%$ and $71.91 \%$, specificity of $89.3 \%$ and $75.25 \%$, PPV of $81.3 \%$ and $71.91 \%$, NPV of $65.8 \%$ and $75.25 \%$ and AUC of 0.793 and 0.792 , respectively $[33,34]$.

Erdal et al. [35] found that DKK-1 alone was less sensitive and specific and had lower PPV and NPV than AFP alone for comparing HCC patients with both cirrhotic patients and controls. DKK-1 alone was not a valid substitute for AFP as a screening test in HCC surveillance. They reported that DKK-1 elevation observed in the HCC group indicates that the combination of DKK-1 and AFP could improve the diagnostic accuracy of the screening.

Our study had some limitations: a small number of patients (only eighty subjects included), advanced cirrhosis, where most patients were $\mathrm{C}$ class of the $\mathrm{CP}$ classification (19 out of 30 cirrhotic HCV patients and 21 out of 30 HCC patients); more patients were needed from both class A and class B to clarify the rule of DKK-1 in both groups, in addition to monocentric design which also needed more stages of $\mathrm{CP}$ class patients to be included in the study.

\section{Conclusions}

DKK-1 showed significantly lower levels in patients with chronic HCV-related liver cirrhosis without HCC and in patients with chronic HCV-related liver cirrhosis with HCC compared to the control. The combination of DKK-1 and AFP could further improve the diagnostic accuracy of HCV-related cirrhosis with or without HCC. Also DKK-1 is a less accurate biomarker for HCC compared to AFP.

\section{Disclosure}

The authors report no conflict of interest.

\section{References}

1. Niehrs C. Function and biological roles of the Dickkopf family of Wnt modulators. Oncogene 2006; 25: 7469-7481.

2. Miyagawa $Y$, Okita $H$, Itagaki M, et al. EWS/ETS regulates the expression of the Dickkopf family in Ewing family tumor cells. PLoS One 2009; 4: e4634.

3. Chen L, Li M, Li Q, et al. DKK1 promotes hepatocellular carcinoma cell migration and invasion through $\beta$-catenin/MMP7 signaling pathway. Mol Cancer 2013; 12: 157.

4. Kolligs FT, Bommer G, Göke B. Wnt/Beta-Catenin/Tcf signaling: a critical pathway in gastrointestinal tumorigenesis. Digestion 2002; 66: 131-144.

5. Begenik H, Kemik A, Emre H, et al. The association between serum Dickkopf-1 levels and esophageal squamous cell carcinoma. Hum Exp Toxicol 2013; 33: 785-788.

6. Dong LL, Qu LY, Chu LY, et al. Serum level of DKK-1 and its prognostic potential in non-small cell lung cancer. Diagn Pathol 2014; 9: 52.

7. Lin C, Wang J, Wang F. Dickkopf-1 promotes hyperglycemia-induced accumulation of mesangial matrix and renal dysfunction. J Am Soc Nephrol 2010; 21: 124-125.

8. Qi L, Sun B, Liu Z, et al. Dickkopf-1 inhibits epithelial-mesenchymal transition of colon cancer cells and contributes to colon cancer suppression. Cancer Sci 2012; 103: 828-835.

9. Abdel-Wahab R, Hassan M, Eid S, et al. The differential clinicopathologic features and survival outcome between hepatitis C-related HCC patients in the United States versus Egypt. J Clin Oncol 2014; 32: e15128.

10. Omar A, Abou-Alfa GK, Khairy A, Omar H. Risk factors for developing hepatocellular carcinoma in Egypt. Chin Clin Oncol 2013; 2 : 43.

11. Heimbach JK, Kulik LM, Finn RS, et al. AASLD guidelines for the treatment of hepatocellular carcinoma. Hepatology 2018; 67: 358-380.

12. Kim SU, Park JH, Kim HS, et al. Serum Dickkopf-1 as a biomarker for the diagnosis of hepatocellular carcinoma. Yonsei Med J 2015; 56: 1296-1306.

13. Puri BK. SPSS in practice: an illustrated guide. 2nd ed. Arnold, London, New York 2002.

14. MacDonald BT, Tamai K, He X. Wnt//-catenin signaling: components, mechanisms, and diseases. Dev Cell 2009; 17: 9-26.

15. Sato N, Yamabuki T, Takano A, et al. Wnt inhibitor Dickkopf-1 as a target for passive cancer immunotherapy. Cancer Rese 2010; 70: 5326-5336.

16. Tung EK, Mak CK, Fatima S, et al. Clinicopathological and prognostic significance of serum and tissue Dickkopf-1 levels in human hepatocellular carcinoma. Liver Int 2011;31: 1494-1504.

17. Chen L, Li M, Li Q, et al. DKK1 promotes hepatocellular carcinoma cell migration and invasion through $\beta$-catenin/MMP7 signaling pathway. Mol Cancer 2013; 12: 157.

18. Jeng JE, Chuang LY, Chuang WL, Tsai JF. Serum Dickkopf-1 as a biomarker for the diagnosis of hepatocellular carcinoma. Chin Clin Oncol 2012; $1: 4$

19. Behne T, Copur MS. Biomarkers for hepatocellular carcinoma. Int J Hepatol 2012; 2012: 859076.

20. Tsuchiya N, Sawada Y, Endo I, et al. Biomarkers for the early diagnosis of hepatocellular carcinoma. World J Gastroenterol 2015; 21: 10573-10578.

21. Wang Q, Chou X, Guan F, et al. Enhanced Wnt signalling in hepatocytes is associated with schistosoma japonicum infection and contributes to liver fibrosis. Sci Rep 2017; 7: 230. 
22. Taylan A, Sari I, Akinci B, et al. Biomarkers and cytokines of bone turnover: extensive evaluation in a cohort of patients with ankylosing spondylitis. BMC Musculoskelet Disord 2012; 13: 191.

23. Han SX, Zhou X, Sui X, et al. Serum Dickkopf-1 is a novel serological biomarker for the diagnosis and prognosis of pancreatic cancer. Oncotarget 2015; 6: 19907-19917.

24. Liu Y, Tang W, Xie L, et al. Prognostic significance of Dickkopf-1 overexpression in solid tumors: a meta-analysis. Tumor Biol 2014; 35: 3145-3154.

25. Ahmed MA, Hassan I, Hassuna N, Darwish A. Significance of Dickkopf-1 (dkk-1) as a biomarker in chronic hepatitis $\mathrm{c}$ and its progression to hepatocellular carcinoma. J Clin Biomed Sci 2014; 44: 1-16.

26. Huang Y, Yang X, Zhao F, et al. Overexpression of Dickkopf-1 predicts poor prognosis for patients with hepatocellular carcinoma after orthotopic liver transplantation by promoting cancer metastasis and recurrence. Med Oncol 2014; 31: 966.

27. Voorzanger-Rousselot N, Goehrig D, Facon T, et al. Platelet is a major contributor to circulating levels of Dickkopf-1: clinical implications in patients with multiple myeloma. Br J Haematol 2009; 145: 264-266.

28. Fouad YM, Mohamed HI, Kamal EM, Rasek MA. Clinical significance and diagnostic value of serum dickkopf- 1 in patients with hepatocellular carcinoma. Scand J Gastroenterol 2016; 51: 1133-1137.

29. Shen Q, Fan J, Yang XR, et al. Serum DKK1 as a protein bioarker for the diagnosis of hepatocellular carcinoma: a large-scale, multicentre study. Lancet Oncol 2012; 13: 817-826.

30. Shi RY, Yang XR, Shen QJ, et al. High expression of Dickkopfrelated protein 1 is related to lymphatic metastasis and indicates poor prognosis in intrahepatic cholangiocarcinoma patients after surgery. Cancer 2013; 119: 993-1003.

31. Qin QF, Weng J, Xu GX, et al. Combination of serum tumor markers dickkopf-1, DCP and AFP for the diagnosis of primary hepatocellular carcinoma. Asian Pac J Trop Dis 2017; 10: 409-413.

32. Yu B, Yang X, Xu Y, et al. Elevated expression of DKK1 is associated with cytoplasmic/nuclear $\beta$-catenin accumulation and poor prognosis in hepatocellular carcinomas. J Hepatol 2009; 50: 948-957.

33. Yang H, Chen GD, Fang F, et al. Dickkopf-1: as a diagnostic and prognostic serum marker for early hepatocellular carcinoma. Int J Biol Markers 2012; 28: 286-297.

34. Zhang J, Zhao Y, Yang Q. Sensitivity and specificity of Dickkopf-1 protein in serum for diagnosing hepatocellular carcinoma: a meta-analysis. Int J Biol Markers 2014; 29: e403-410.

35. Erdal H, Utku ÖG, Karatay E, et al. Combination of DKK1 and AFP improves diagnostic accuracy of hepatocellular carcinoma compared with either marker alone. Turk J Gastroenterol 2016; 27: $375-381$ 